\title{
A NOTE ON ALMOST PERIODIC SOLUTIONS OF LINEAR PARTIAL DIFFERENTIAL EQUATIONS
}

\author{
BY GEORGE R. SELL ${ }^{1}$
}

Communicated by James Serrin, August 21, 1972

In this note we will present two results concerning the question of existence of almost periodic solutions of the system of linear partial differential equations

$$
\sum_{j=1}^{n} L_{i j} u_{j}=f_{i}, \quad 1 \leqq i \leqq n,
$$

on $R^{m}$, where $L_{i}$ is an arbitrary linear partial differential operator on $R^{m}$ given by

$$
L_{i j}=\sum_{\alpha} a_{\alpha i j} D^{\alpha}
$$

and the summation is finite. (We use the standard notation for partial differential operators, cf. [2] for example.) It will be more convenient to write the system (1) in the form

$$
L u=f,
$$

where $u$ and $f$ are now viewed as mappings of $R^{m}$ into $R^{n}$. The order $k$ of $L$ is defined to be the maximum of the orders of the $L_{i}$.

We will assume that the coefficients $a_{\alpha i j}$ and $f_{i}$ are continuous and almost periodic functions of $t=\left(t^{1}, \ldots, t^{m}\right)$ in $R^{m}$. Recall that $g$ is an almost periodic function of $t$ in $R^{m}$ if, for every sequence $\beta^{\prime}=\left\{\beta_{n}^{\prime}\right\}$ in $R^{m}$, there is a subsequence $\beta=\left\{\beta_{n}\right\}$ such that $\lim g\left(t+\beta_{n}\right)$ converges uniformly for $t$ in $R^{m}$. This notion of almost periodicity, which is due to Bochner for the case $R^{m}=R^{1}$, is equivalent to the Bohr concept of almost periodicity, which is defined in terms of a relatively dense set of translation numbers.

We define the hull $H(L, f)$ to be the collection of all linear partial differential equations $L^{*} u=f^{*}$ where the coefficients $a_{\alpha i j}^{*}$ and $f_{i}^{*}$ are related to $a_{\alpha i j}$ and $f_{i}$ by

(3) $\lim a_{\alpha i j}\left(t+\beta_{n}\right)=a_{\alpha i j}^{*}(t)$ and $\lim f_{i}\left(t+\beta_{n}\right)=f_{i}^{*}(t), \quad t \in R^{x}$,

AMS (MOS) subject classifications (1970). Primary 35B15, 35G05, 35J15, 42A84, 54H20; Secondary 31B35, 34C25, 35D99.

Key words and phrases. Almost periodic functions, bounded solutions, linear partial differential operators.

${ }_{1}$ This work was done while the author was visiting the Istituto Matematico dell' Universita di Firenze under the auspices of the Italian Research Council (C.N.R.). Partial support for this research was also given by NSF grant GP-27275. 
for some sequence $\beta=\left\{\beta_{n}\right\}$, which is independent of $\alpha, i$ and $j$. Since $a_{\alpha i j}$ is assumed to be almost periodic, the limit in (3) is in fact uniform for all $t$ in $R^{m}$, although we shall only require pointwise convergence in (3) to define the hull. $H(L, 0)$ will denote the hull of the homogeneous equation $L u=0$.

A solution $u$ of equation (2) is said to be $C^{k}$-bounded if $u=\left(u_{1}, \ldots, u_{n}\right)$ together with all derivatives up to and including order $k$ are bounded and uniformly continuous on $R^{m}$.

Our first theorem is concerned with classical solutions of equation (2).

THEOREM 1. Let $L$ be a linear partial differential operator of order $k$ and assume that the coefficients $a_{\alpha i j}$ and $f_{i}$ are almost periodic functions of $t$ in $R^{m}$. Assume further that every $C^{k}$-bounded solution of every homogeneous equation $L^{*} v=0$ in the hull $H(L, 0)$ is almost periodic. If $u$ is a $C^{k}$-bounded solution of any equation $L^{*} u=f^{*}$ in the hull $H(L, f)$, then $u$ is almost periodic.

This theorem generalizes to partial differential equations similar results of Favard [3] and Bochner [1] for ordinary differential equations. The proof of this theorem, which will be given elsewhere, follows essentially the argument of Bochner, with appropriate modifications for the case of partial differential equations.

The concept of $C^{k}$-boundedness requires that the highest order derivative be uniformly continuous as well as bounded. In certain special cases one can prove that boundedness implies uniform continuity; for example, this is true for ordinary differential equations. It is also true for Poisson's equation $\Delta u=f$, provided one uses the concept of a generalized solution (cf. [2] and [4]).

The second problem we study in this note is the question of extending Theorem 1 to include generalized solutions. In particular, we study the behavior of bounded solutions of weakly coupled systems of the form

$$
\Delta u_{i}+\sum_{j=1}^{n} a_{i j} u_{j}=f_{i}, \quad 1 \leqq i \leqq n
$$

where $\Delta$ is the Laplacian operator and the coefficients $a_{i j}$ and $f_{i}$ are almost periodic functions of $t$ in $R^{m}$. Once again it is more convenient to write equation (4) in the form

$$
\Delta u+A u=f
$$

The hull of equation (5) is defined to be the collection $H(A, f)$ of all partial differential equations

$$
\Delta u+A^{*} u=f^{*}
$$


where the coefficients $A^{*}$ and $f^{*}$ are related to $A$ and $f$ by

$$
A^{*}(t)=\lim A\left(t+\beta_{n}\right) \quad \text { and } \quad f^{*}(t)=\lim f\left(t+\beta_{n}\right), \quad t \in R^{m},
$$

for some sequence $\beta=\left\{\beta_{n}\right\}$ in $R^{m}$. For the homogeneous equation $\Delta v+A v=0$, the hull is simply $H(A, 0)$.

We define a generalized solution of equation (5) to be a continuous function $u: R^{m} \rightarrow R^{n}$ with the property that, for every $C^{\infty}$-function $\phi: R^{m} \rightarrow R^{n}$ with compact support, one has

$$
\int_{R_{m}}[(u, \Delta \phi)+(A u, \phi)] d t=\int_{R_{m}}(f, \phi) d t .
$$

We use $(u, u)$ to denote an inner product on $R^{n}$ and let $|u|=(u, u)^{1 / 2}$ be the induced norm.

For equation (5) we can prove the following result:

THEOREM 2. (A) Let $A$ and $f$ be almost periodic functions of $t$ in $R^{m}$. Assume that if $v$ is any nontrivial bounded generalized solution of any homogeneous equation $\Delta v+A^{*} v=0$ in the hull $H(A, 0)$, then

$$
\inf \left\{|v(t)|: t \in R^{m}\right\}>0 \text {. }
$$

If there exists a bounded generalized solution $u$ of equation (5), then there exists an almost periodic generalized solution $\tilde{u}$ of equation (5).

(B) If every bounded generalized solution of every homogeneous equation in the hull $H(A, 0)$ is almost periodic, then every bounded generalized solution of equation (5) is almost periodic.

Theorem 2 includes as a special case the theorem of Sibuya for Poisson's equation, which one gets by setting $n=1$ and $A=0$ (cf. [4]). In this case, the only bounded generalized solutions of the homogeneous equation $\Delta u=0$ are constant functions.

Also if one sets $m=1$ and replaces $\Delta$ with the ordinary differential operator $D$, where $D u_{i}=d u_{i} / d t$, then our theorem includes the wellknown result of Favard [3, pp. 90-94] for ordinary differential equations.

\section{REFERENCES}

1. S. Bochner, A new approach to almost periodicity, Proc. Nat. Acad. Sci. U.S.A. 48 (1962), 2039-2043. MR $26 \# 2816$.

2. R. Courant, Methods of mathematical physics. Vol. II. Partial differential equations, Interscience, New York, 1962. MR 25 \# 4216.

3. J. Favard, Leçons sur les fonctions presque-périodiques, Gauthier-Villars, Paris, 1933.

4. Y. Sibuya, Almost periodic solutions of Poisson's equation, Proc. Amer. Math. Soc. 28 (1971), 195-198.

Department of Mathematics, University of Minnesota, Minneapolis, Minnesota 55455 